\title{
How Can We Meet the Needs of Young People in Vulnerable 'NEET' Situations? A Realist Research Protocol to Evaluate the Delivery and Implementation of $(\mathrm{Re})$ Engagement Initiatives in Northern Sweden
}

Frida Jonsson ( $\square$ frida.jonsson@umu.se )

Umeå Universitet Medicinska fakulteten: Umea Universitet Medicinska fakulteten https://orcid.org/0000-0002-5902-3798

Jan Hjelte

Umeå Universitet

Linda Richter Sundberg

Umeå Universitet Medicinska fakulteten: Umea Universitet Medicinska fakulteten

Annika Nordström

Umeå Universitet Medicinska fakulteten: Umea Universitet Medicinska fakulteten

Isabel Goicolea

Umeå Universitet Medicinska fakulteten: Umea Universitet Medicinska fakulteten

Study protocol

Keywords: northern Sweden, NEET, young people, programmes, realist evaluation, concept mapping

Posted Date: June 2nd, 2021

DOl: https://doi.org/10.21203/rs.3.rs-544387/v1

License: (c) (i) This work is licensed under a Creative Commons Attribution 4.0 International License.

Read Full License 


\section{Abstract \\ Background}

Situated in the northern Swedish context, the project presented in this protocol aims to explore and establish how multi-component '(re)engagement' initiatives work, while providing directions for strengthening the delivery and implementation to better meet the needs of young people in vulnerable 'NEET' situations ('not in employment, education or training'). In this regard, the research emerges from international and national concerns about the challenges faced by young people who are marginalised from mainstream institutions. It is also a response to the '(re)engagement' initiatives that have been implemented, but never rigorously evaluated, in Sweden, which means a substantial body of relevant knowledge is at risk of being lost, and that we currently lack evidence about what works to reengage young people that are neither studying nor working.

\section{Methods}

To address the aim and three subsequent research questions, two methodologies will be combined in three phases. Following an iterative analytical process, where results from a realist evaluation will inform a concept mapping study, the project will involve: 1) a realist review of international academic and grey literature to identify underlying programme theories and explore how '(re)engagement' initiatives are expected to work; 2 ) a realism-based case study to scrutinise the programme theories and assess whether the '(re)engagement' initiatives work as intended; and 3) a participatory concept mapping study to provide directions for strengthening the delivery and implementation of '(re)engagement' initiatives in northern Sweden.

\section{Discussion}

While '(re)engagement' initiatives may not be a solution to the problems that exist in labour markets and educational systems today, they may be a partial and perhaps provisional saviour for young people who have already ended up at the margins of society. By evaluating how these initiatives work while providing directions for action, the current project will contribute knowledge useful to improve the support to young people in vulnerable 'NEET' situations who need the courage to dare enter a world that has let them down once before.

\section{Contributions To The Literature}

- Moving beyond the question of 'does it work', this project will fill a knowledge gap by identifying ways though which '(re)engagement' initiatives can meet the needs of young people in vulnerable 'NEET' situations. 
- Related to a lack of scientific attention paid to the integration of these initiatives in Sweden and beyond, the project will add to research and practice by assessing how the delivery and implementation can be strengthened.

- The combination of realist evaluation and concept mapping is not only useful to generate findings that will be relevant for policy and practice, it is also an innovative approach that provides opportunities for methodological advancements.

\section{Introduction}

The situation of young people who are in not employment, education, or training - usually referred to in policy, public, and academic discourses as 'NEETs' - has received political and societal attention across Europe [1], including Sweden [2] for at least a decade. Conceptually, the abbreviated term 'NEET' is typically used to categorise unemployed young people aged 15 to 29 or 34 years who seek work, and inactive non-students who officially neither hold nor look for a job [3]. While this means that the group may include those who enjoy a number of privileges and choose not to engage in education or employment, in the current project we recognise discussions about the broad stroke classification of 'NEETs' [4] by limiting our scope to segments of this population that, due to various constraints, lack options and opportunities in life.

According to the most recent data from Eurostat [5], the 'NEET' rate varies greatly across Europe, with levels ranging from about $7-11 \%$ in the Nordic countries, including Sweden, to about $20-25 \%$ among southern countries, such as Italy, Greece, and Serbia. In light of the ongoing COVID-19 pandemic, the challenges facing this group of young people, more generally, and certain sub-groups, in particular, have become increasingly apparent. Research [6] and youth organisations [7] internationally have thus called for immediate policy action to avoid losing a generation due to the potential long-term consequences of experiencing periods of unemployment during youth and young adulthood [8]. In this regard, all member states in the European Union recently committed to the implementation of a reinforced Youth Guarantee [9]. However, by relying heavily on national public employment services to facilitate the transition of 'NEETs' into education or employment, research evaluating an earlier version shows inconsistent results. Specifically, while the guarantee appears to slightly benefit young people who stand quite close to the labour market, it appears to be insufficient to support sub-groups in more vulnerable situations $[10,11]$.

To create real and sustained change for young people whose educational trajectories, social circumstances, and economic conditions are particularly challenging [12], moving beyond standardised solutions characterised by a premature and largely instrumental focus on getting them to study or work will be essential [13]. In particular, meeting the needs of 'NEETs' who face substantial life barriers due, for example, to long-term unemployment, chronic or mental illness, disability, alienation, or discouragement [14] may require more flexible, holistic, and indeed humane approaches [15]. To contribute insights about the workings of such efforts, this protocol presents a project that will evaluate and provide directions for strengthening the delivery and implementation of multi-component '(re)engagement' initiatives, 
introduced in northern Sweden to the (potential) benefit of young people in vulnerable 'NEET' situations [16].

\section{Scope and aim of the project}

Beyond being concerned with various behavioural and health-related outcomes, increasing the likelihood of 'NEETs' finding meaningful work and engaging in decent jobs by strengthening their employment and educational readiness, typically constitutes the overarching goal of '(re)engagement' initiatives $[13,17]$. To facilitate this scope, isolated strategies like one-to-one adult-youth mentoring [18], life skill development through sports and physical activity [19], and academic or work-based learning and training [20] have so far been adopted across Europe and beyond. However, being limited in scope, reports have suggested that such singular efforts may be insufficient to meet the complex needs of young people in more vulnerable 'NEET' situations, while initiatives that combine different resources may resonate better with their varied interests, talents, and abilities [21, 22].

Overall, the literature has described how multi-component '(re)engagement' initiatives place an emphasis on personalised and applied learning, practical work experience and skills for social living, whereby professionals offer a range of tailored activities and forms of therapeutic support to the young people [23]. In the Swedish context, these efforts have been defined according to their provision of skill-building and health-promoting activities in combination with forms of holistic, personalised, and coordinated support delivered by authentic, caring, and compassionate adults [16]. Considering the complexity of these initiatives, the potential pathways to success are numerous. However, due to a concurrent focus on their effectiveness or efficacy to reduce youth unemployment, we currently lack insights into and evidence about how, and why, they work [21].

To address these knowledge gaps, the theory-driven realist evaluation methodology, which aims to explore and establish 'what works, for whom and in what contexts', may be a useful approach $[24,25]$. To the best of our knowledge, only two theory-driven evaluations have assessed the role of multi-component '(re)engagement' initiatives for young people in vulnerable 'NEET' situations. In England, a realist evaluation of the nation-wide, now largely dismantled, Connexions service was carried out in 2004 [26]. In Wales, Owen [27] more recently evaluated both the school-based TACKLE programme and the youthdriven EMPOWER programme. Regarding the latter assessment, the study showed (amongst other things) that when young people that are in 'NEET' situations are given the opportunity to participate in captivating activities where they have to be present in the moment, mechanisms of engagement and focus are activated. This, in turn, allows them to regulate their emotions and attention (i.e., become focused, mindful, and calm), thus engaging successfully to complete the task at hand.

In contrast to the UK-based efforts evaluated to date, the type of initiatives at the centre of our project does not follow a specified, time-limited format carried out, for example, within the school setting, and neither are the initiatives part of a coherent national establishment. Instead, through what appears to be an organic and highly innovative process, '(re)engagement' initiatives in both rural and urban parts of 
northern Sweden have (at least for now) developed from, and to meet, a locally identified need. Despite this bottom-up and community-based perspective, the different initiatives, which are typically run and funded by the local municipality, share many similarities in the type of activities and support they provide young people in vulnerable 'NEET' situations, as illustrated in our previous research [16].

\section{Project aim}

By integrating the realist evaluation [24] and concept mapping [28] approaches (see details below), the project presented in this protocol aims to explore and establish how northern Swedish '(re)engagement' initiatives work, while providing directions for strengthening the delivery and implementation to better meet the needs of young people in vulnerable 'NEET' situations. In this regard, the research is a response to the call for rigorous, theoretically driven evaluations, which recognises the value of local innovation and of identifying nuances in service delivery occurring between sites [21]. With a subsequent focus on generating evidence useful for policy and practice by identifying what works to reengage young people, the project will address the following research questions:

1. How are '(re)engagement' initiatives in northern Sweden expected to work?

2. How are '(re)engagement' initiatives in northern Sweden actually working?

3. How can the practices and processes of '(re)engagement' initiatives in northern Sweden be strengthened?

\section{Methodologies And Design}

See additional file 1 for the populated reporting checklist (RAMESES II reporting standards for realist evaluations [25]) that guided our development of this protocol article.

\section{Setting}

In Sweden overall, the government structure is highly decentralised, including two sub-national levels comprising 21 regions (responsible for health care) and 290 municipalities (responsible, e.g., for social, elderly, and childcare), with a considerable degree of autonomy and independent powers of taxation. This form of self-governing provides opportunities for flexible, innovative, and context-specific solutions, useful to develop and deliver services in accordance with local needs. In this project, our research will be situated in the four northernmost regions of Sweden (Norrbotten, Västerbotten, Västernorrland, and Jämtland/Härjedalen). Together, these cover about $60 \%$ of the Swedish land, while being home to only about $12 \%$ of the total population residing in 44 municipalities. As such, this is a sparsely populated area, where people live in small rural villages inland or in somewhat larger urban cities along the coast.

In this area, the main providers of health and social care to young people are primary health centres, specialised child psychiatry units, school health, youth clinics, and youth clubs. Education in Sweden is free of charge and compulsory for nine years between ages 6 and 15/16. Upper-secondary school of three years is optional and includes six national programmes that are preparatory for higher education 
and 12 that are vocational. Out of the $86 \%$ of Swedish ninth-grade students who qualified for a national programme in 2020, about $72 \%$ finalised their studies [29]. Higher education in Sweden is publicly funded, with tuition fees being fully subsidised for all national and EU/EEA (including Switzerland) citizens. In contrast to many other countries, the Swedish system for higher education is fairly flexible by offering good opportunities for lifelong learning. For young people in general, this means that a relatively low percentage enter into higher education directly after upper-secondary school, with many new entrants being 25 years or older [30].

\section{Methodological approaches}

To explore and establish how northern Swedish '(re)engagement' initiatives work while providing directions for strengthening their delivery and implementation to better meet the needs of young people in vulnerable 'NEET' situations, the current project will combine the realist evaluation [24] and concept mapping [28] approaches in three phases.

\section{Realist evaluation}

We will start by conducting a realist evaluation to address the first two research questions. Realist evaluation is a theory-driven research strategy with origins in scientific realism that is concerned not only with whether an initiative (i.e., intervention, policy, programme, or service) manages to meet its objectives but with how it works, for whom, in what conditions, and why [24]. Central to this approach is the idea that socially contingent initiatives are underpinned in design and functioning by explicit or implicit theories comprising assumptions about ways through which effects occur [25].

When conducting a realist evaluation, a defining initial step is to elicit these underlying programme theories by mapping, developing, and, ultimately, converting theorised causal claims into testable hypotheses. To facilitate this scope, information from diverse sources such as hunches, previous knowledge, the literature, retroductive thinking, and stakeholder consultations is conceptualised into context-mechanism-outcome (CMO) configurations, which constitute the analytical units of realist inquiries [31]. Based on the need of evaluators to 'battle with' complex realities [24], the CMO configuration is a heuristic used in a realist evaluation (or synthesis) to generate causal statements that link mechanisms with aspects of context and outcomes.

Defined as the underpinning causal pathways with the power to generate outcomes (i.e., intended or unintended effects or outputs of the programme) in a given context, from a realist perspective, mechanisms pertain to the psychosocial reasoning and reactions of people in response to resources and opportunities offered by the programme [32-34]. Context, in turn, captures conditions occurring before, or existing outside of, the programme with the potential to activate the mechanisms during implementation [35]. Building on these definitions, the $\mathrm{CMO}$ heuristic offers a pragmatic approach to programme complexity. However, beyond providing a structure for configuring ' $C s^{\prime}$ ', 'Ms', and 'Os', it explicates the philosophical underpinnings of scientific realism, which state that explanations to empirically manifest and measurable outcomes emerge from dynamic interactions between contextual factors and potentially 
hidden or dormant mechanisms of action. By being real, but most likely not directly visible, these latter generative mechanisms can be unearthed only through retroduction because they exist in the ontologically deep layers of reality [36].

Once the initial programme theories have been constructed, then they are scrutinised against primary data (information collected, e.g., through interviews, observations, and literature) to be confirmed, refuted, or refined to assess whether and how theory and practice correspond [37].

\section{Concept mapping}

Based on findings from the realist evaluation, we will conduct a concept mapping study [28] to address the third research question. By giving participants the opportunity to share their knowledge and discuss an issue of mutual interest, this approach has been considered useful for developing culturally tailored programmes and to increase community involvement in research [38].

As explained by Kane and Trochim [28], concept mapping involves the generation and integration of qualitative and quantitative data by participants in sequential steps. After engaging key stakeholders in what will ultimately be a structured and participatory process, the work begins with the generation of ideas in a brainstorming where participants are asked to answer a 'prompt' or 'focused' question that is often visionary, aiming to identify areas for improvements. From this process emerges a large number of ideas that, in a second step, are sorted and rated by the participants in ways that appear meaningful to them. Once organised into clusters and ranked according to some chosen indicators (e.g. relevance and feasibility) the ideas are then translated into pictorial form and developed into conceptual maps using multivariate statistical methods. In a final consensus meeting, the findings are then interpreted by the team of researchers in dialogue with the participants and other stakeholders to identify and propose directions for action.

\section{Participants}

As discussed by Pawson and Tilley [31], the purposive inclusion of participants in the scrutinising phase of a realist evaluation should be guided by theoretical considerations, according to their ' $\mathrm{CMO}$ investigation potential'. As such, rather than aiming for balance or consensus, the goal should be to capture a range of understandings, since the programme dynamics can be experienced differently by different people [39]. In this regard, participants in our project will broadly and tentatively include service managers (i.e., professionals with authority to make decisions, such as project leaders), frontline workers (i.e., practitioners, such as social workers, counsellors, and special educators), and, most importantly, currently enrolled and alumni young people.

\section{Overall study design}


Table 1

Outline of the research process

presents an overview of the research process, with each phase explained in more detail below.

\begin{tabular}{|c|c|c|c|}
\hline Methodology & Phase and research question & Steps & Methods \\
\hline \multirow{4}{*}{$\begin{array}{l}\text { Realist } \\
\text { evaluation }\end{array}$} & Phase 1 & \multirow{2}{*}{$\begin{array}{l}\text { Contextualise and } \\
\text { elicit the programme } \\
\text { theories }\end{array}$} & \multirow{2}{*}{$\begin{array}{l}\text { Realist } \\
\text { synthesis }\end{array}$} \\
\hline & $\begin{array}{l}\text { How are (re)engagement initiatives in } \\
\text { northern Sweden expected to work? }\end{array}$ & & \\
\hline & Phase 2 & \multirow{2}{*}{$\begin{array}{l}\text { Scrutinise and refine } \\
\text { the programme } \\
\text { theories }\end{array}$} & \multirow{2}{*}{$\begin{array}{l}\text { Multiple case } \\
\text { study }\end{array}$} \\
\hline & $\begin{array}{l}\text { How are (re)engagement initiatives in } \\
\text { northern Sweden actually working? }\end{array}$ & & \\
\hline \multirow{3}{*}{$\begin{array}{l}\text { Concept } \\
\text { mapping }\end{array}$} & Phase 3 & Generation of ideas & Brainstorming \\
\hline & \multirow[t]{2}{*}{$\begin{array}{l}\text { How can the practices and processes of } \\
\text { Swedish (re)engagement initiatives be } \\
\text { strengthened? }\end{array}$} & $\begin{array}{l}\text { Structuring ideas } \\
\text { and developing } \\
\text { concept maps }\end{array}$ & $\begin{array}{l}\text { Sorting, } \\
\text { rating, and } \\
\text { visualisation }\end{array}$ \\
\hline & & $\begin{array}{l}\text { Synthesising ideas } \\
\text { and proposing } \\
\text { actions }\end{array}$ & Interpretation \\
\hline
\end{tabular}

To address the first research question (see Table 1), we will conduct a realist synthesis [40] of international academic and grey literature.

\section{Realist synthesis}

Extending beyond standard systematic reviews focused on the question of 'does it work', the ambition of a realist synthesis is to assess how it works, for whom, in what conditions, and why [41]. To facilitate this scope, the analytical process involves articulating initial programme theories, which are then interrogated against existing evidence to 'find out whether and where these theories are pertinent and productive' [42 $\mathrm{p}$. 74]. This means that a realist synthesis often begins with informal discussions and exploratory readings of key references to theorise potentially relevant programme theories and locate middle-range theories useful to conceptualise and permit empirical testing of the causal claims. Testing all the identified ideas, assumptions, and rationales is often impossible. The long list of theories is therefore inspected to prioritise the ones that should be scrutinised against existing evidence (i.e., the literature) [40].

Conducting a realist synthesis of international academic and grey literature as a first step of our project will allow us to explore the programme theories underlying '(re)engagement' initiatives directed towards young people in vulnerable 'NEET' situations, thus increasing our 'theoretical sensitivity' in the area. By testing the theories against existing evidence, we will also gain insights into how the initiatives work in 
practice - knowledge that we will apply and re-evaluate in the Swedish setting. However, before scrutinising the programme theories in the second step (see below), following Saul et al. [43] we will engage in discussions with a local reference group to make sure the review findings are relevant for the northern Swedish context. To ensure scientific rigour, the synthesis will be conducted following the structure and standards outlined in the literature $[44,45]$.

\section{Phase 2: Scrutinise and refine the programme theories}

To address the second research question (see Table 1), we will scrutinise and refine the programme theories developed from the realist synthesis and discussions with local stakeholders against empirical data [24]. To facilitate this scope, a realism-based case study approach [46] will be combined with the realist interview technique for data collection $[39,47]$ and the realist approach to thematic analysis for analysing the data [48].

According to Wynn and Williams [46], realism-based case studies are especially suitable for scrutinising and refining programme theories. Firstly, the approach provides opportunities for detailed narratives that explicate causal mechanisms while exposing important contextual elements (e.g., structure and culture) - to be developed, for example, through retroduction by observation logging and memos [see e.g. 49]. Secondly, they provide a solid base for answering questions about how and why change or effects have occurred [50].

As a complement to the centrality of participant observations in realism-based case studies [46], we will conduct semi-structured realist interviews [39] using vignettes as a teacher-learner tool [51] following the 'I'll show you my theory, if you'll show me yours' approach to data collection [31]. From this perspective, the craft of realist interviewing tends to follow a 'teacher-learner' cycle, where the interviewer and the interviewee exchange ideas in a dynamic conversation of thinking through the programme complexities [39]. Rather than simply capturing the participants' thoughts about the theories overall, the aim is to discern in dialogue with, and based on, their experiences what aspects of the $\mathrm{CMO}$ configurations under test are applicable and accurate. In this regard, the theories will be the subject matter of the interview, and with the subject being there 'to confirm or falsify and, above all, to refine' the theories [47 p. 299].

Besides developing thick descriptions as part of the case study approach, which will involve looking into and sorting out the rich details of each case [52], data from detailed observation memos and transcribed interviews will be analysed using the novel realist approach to thematic analysis [48]. Since the aim of a realist analysis is to gain generative causal insights about the underpinning mechanisms that contribute to programme success, and increase our ontologically deep understanding of the studied phenomena [36], the analytical process will involve exploring pieces of data (or 'fragments' [see 53]) to paint a causal picture useful to reformulate the programme theories. Conducting a realist thematic analysis, which provides opportunities to accurately capture both the empirical world (through experiential themes) and the potential powers that exist in the deeper layers of reality (through inferential and dispositional themes), will be especially useful [48]. 
In this project, five to seven '(re)engagement' initiatives located in different rural and urban parts of northern Sweden will be purposively selected as our 'cases' to provide a basis to empirically examine the initial programme theories. To collect data for scrutinising and refining the theories, in each case we will conduct i) realist interviews [39] with young people, frontline workers, and managers; ii) participatory observations of the daily work [54] and iii) document reviews as appropriate [55]. As explained by Manzano [39], the realist process of theory testing is (and should be) unpredictable, unstable, and uncertain to allow for new issues to emerge and be iteratively integrated through the research. As such, the number of participants involved in our research will neither be decided a priori nor be guided by saturation but will follow from an iterative data collection process aimed at achieving sufficient variability to 'move from constructions to explanation of causal mechanisms' [39 p. 348].

\section{Phase 3: Identifying practices, processes and directions for action}

To address the third research question (see Table 1), knowledge gained during the previous phases will be used to engage young people in vulnerable 'NEET' situations and relevant stakeholders in a concept mapping study [28].

Based on the sequential steps of concept mapping [35], we will start this last phase with a series of workshops, separately with the young people and with the stakeholders, to engage potential participants and present findings from the realist evaluation. In this regard, the workshops will function as a catalyst for the coming steps and involve a brainstorming where participants will be asked to discuss ideas on a) service adaptations that could promote dissemination and b) tailored implementation strategies that could promote integration and uptake. After the workshops, all participants will be invited to participate in a sorting and rating activity conducted using the 'GroupWisdom' software [56]. This approach will allow us to analyse the brainstorming data and develop conceptual maps to visualise thematic clusters. In a final step, separate workshops with the young people and stakeholders will be conducted to discuss the maps and preliminary findings. The aim will be to find consensus areas for action and identify practices and processes useful to strengthen the delivery and implementation of Swedish (re)engagement initiatives to better meet the needs of young people in vulnerable 'NEET' situations.

\section{Ethical considerations}

The Swedish Ethical Review Authority (Dnr 2021 - 01418) has approved the current project, and in accordance with the Declaration of Helsinki [57], the upcoming studies pay attention to the needs and priorities of young people faced with substantial barriers that so far have been underrepresented in research. Protecting the participants' privacy and the confidentiality of their personal information while minimising the impact of the research on their physical, mental, and social integrity will lie at the heart of the project. To ensure these commitments are met, we will seek informed consent (written and verbal) from each participant in a reflexive way by continuously discussing and informing them about the project and its implications and explaining that taking part is voluntary and that they can opt out at any time without giving a reason. We will also ensure that the participants' identities are protected and that 
personal or sensitive information cannot be tied to a specific individual by only working with coded data and by using pseudonyms for people and places when publishing and presenting the findings.

Beyond the above standard approaches, doing research 'with' as compared to 'on' young people in vulnerable 'NEET' situations requires specific ethical considerations $[58,59]$. To gain trust from and promote participation of a group that may be both physically and emotionally 'hard to reach' [60], we will work to level unequal power relations and disrupt, to the extent possible, the adult/young person and researcher/researched subject positions. By familiarising ourselves extensively with the setting and striving to establish collaborative partnerships that are respectful, emancipatory, and focused on a coconstruction of knowledge our hope is that this will be at least partly achievable. In this regard, prolonged engagement involving repeated, close, clear, and unambiguous communication with the young people before, during, and after the data collection will be essential [58]. To ensure that the young people gain some ownership of the process and reduce constraints arising from unfamiliar social situations, we will also let the participants decide how (i.e., more 'traditional' realist interviews in isolation or coupled with, for example, drawing or sentence completion tasks) [59] and where the data collection should take place [58].

\section{Discussion}

In this article, we present a protocol of a research project where an initial realist evaluation [24] will be conducted to explore and establish how '(re)engagement' initiatives work in northern Swedish rural and urban contexts. To provide directions for strengthening the delivery and implementation of these efforts to better meet the diverse and complex needs of young people in vulnerable 'NEET' situations, a concept mapping study [28] will be carried out in a final phase to complement the evaluation results. In this regard, the current project has the potential to develop knowledge useful for policy and practice to make a difference in the lives of young people who are marginalised from mainstream education and employment [13].

The starting point of this research was an earlier project of ours [61], in which we explored what characterises landscapes of care for rural youth [62]. To facilitate this scope and integrate the diverse voices of young people living in rural northern Sweden, several young people in vulnerable 'NEET' situations participated in our studies. Like other stories portrayed in the literature [see, e.g., 63], the young people we met articulated a discomfort and even pain of being perceived as 'different' and not fitting in, and of wanting to stand on their own two feet and thus trying repeatedly to meet societal expectations while blaming themselves when failing. Through reports of how provision of practical and emotional support gave them a sense of direction in life, the young people also allowed us to see first-hand the important role that '(re)engagement' initiatives played in their lives. Inspired by these sad yet hopeful stories, we developed and received funding for the research to be conducted in the current project.

Beyond the potential benefits of our project, and of '(re)engagement' initiatives more generally to make a substantive and sustainable difference in the lives of young people in vulnerable 'NEET' situations, the 
broader context in which research and practice play out needs to be recognised. In this regard, we share the view of many scholars who before us have problematised the development of efforts aimed at reengaging young people by depicting how they tend to be a result of social inequalities, an individualisation of risks, and a broken welfare system that fails to account for everyone by deeming some groups unfit to 'fit in' $[15,64]$. More specifically, while the initiatives at the centre of our project may be a way to interact with marginalised young people in a more humane and less instrumental manner, they should not be seen as places from which these youth can escape alienation [65]; neither do they address the problems in labour markets and education systems today, thus generally letting 'the mainstream off the hook' [15 p. 493].

To find answers to young people's disadvantage, political, public, and academic debates must move beyond conventional deficit explanations of personal barriers and direct attention instead to the broader social and institutional structures that have excluded them in the first place [66]. While such a shift will be essential to prevent young people from ever ending up in a 'NEET' situation, existing '(re)engagement' initiatives may be a partial solution and (perhaps) a provisional saviour for those who have already been abandoned by many parts of mainstream society [64]. Exploring the impact of these efforts and their potential to support young people in vulnerable 'NEET' situations to develop the courage to dare enter a world that has let them down once before is the goal of the current project.

\section{Declarations}

\section{Ethics approval and consent to participate}

The protection of the rights, privacy and integrity of the participants will be crucial throughout the entire study. Before giving their written informed consent, participants were informed about the study and its implications, and that taking part was voluntary. This research has been performed in accordance with the Declaration of Helsinki and approved by the Swedish Ethical Review Authority (Dnr 2021-01418).

\section{Consent for publication}

N/A

\section{Availability of data and materials}

N/A

\section{Competing interests}

The authors declare that they have no competing interests. 


\section{Funding}

This study was supported by the Swedish Research Council for Health, Working life and Welfare (Forte) under Grant [2020-01339]. The funding bodies had no further involvement in the research process.

\section{Authors' contributions}

FJ is the principal investigator of the project. She developed the idea, designed, and secured funding for the research in collaboration with JH, LRS, AN and IG. All authors provided input into various aspects of the study design and revised drafts of the protocol. FJ led the writing of this protocol manuscript. All authors read and approved the final manuscript.

\section{Acknowledgements}

$\mathrm{N} / \mathrm{A}$

\section{References}

1. Eurofound. NEETs - Young people not in employment, education or training. Luxembourg: Publications Office of the European Union; 2012.

2. Ministry of Education. Utveckling av arbetet med unga som varken arbetar eller studerar. Stockholm: Ministry of Education; 2012.

3. Elder S. What does NEETs mean and why is the concept so easily misinterpreted? Work 4 Youth Technical Brief No.1. Geneva: ILO Publishing; 2015.

4. Furlong A. Not a very NEET solution: representing problematic labour market transitions among early school-leavers. Work, Employment and Society. 2006; doi: 10.1177/0950017006067001

5. Eurostat. Young people neither in employment nor in education and training by sex, age and degree of urbanisation (NEET rates) 2020 [updated 21-04-2021. Available from: https://appsso.eurostat.ec.europa.eu/nui/show.do?dataset=edat_lfse_20\&lang=en.

6. Tamesberger D, Bacher J. COVID-19 Crisis: How to Avoid a 'Lost Generation'. Inter economics. 2020; doi: 10.1007/s10272-020-0908-y.

7. OECD. Youth and Covid-19: Response, recovery and resilience. Paris: OECD; 2020.

8. Scarpetta S, Sonnet A, Manfredi T. Rising Youth Unemployment During The Crisis. OECD Social, Employment and Migration Working Papers, No 106. 2010. doi: 10.1787/5kmh79zb2mmv-en.

9. European Commission. Proposal for a Council Recommendation on A Bridge to Jobs - Reinforcing the Youth Guarantee. Brussels: European Commission; 2020.

10. Hämäläinen K, Hamalainen U, Tuomala J. The Labour Market Impacts of a Youth Guarantee: Lessons for Europe? : Government Institute for Economic Research Working Papers No. 60; 2015. 
11. Mascherini M. Youth Guarantee: Experiences from Finland and Sweden. Loughlinstown, Dublin: Eurofound; 2012.

12. Plenty SB, Andersson A, Hjalmarsson S, Mood C, Rudolphi F, Treuter G. How are our young adults doing? A report on labour market activities and living conditions. Stockholm: The Institute for Future Studies; 2018:1.

13. Yates S, Payne M. Not so NEET? A Critique of the Use of 'NEET' in Setting Targets for Interventions with Young People. Journal of Youth Studies. 2006; doi: 10.1080/13676260600805671.

14. Eurofound. Exploring the diversity of NEETs. Luxembourg: Publications Office of the European Union; 2016.

15. Smyth J, Robinson J, McInerney P. It's our turn - young people 'tilting' the neoliberal turn. Journal of Youth Studies. 2014; doi: 10.1080/13676261.2013.830705.

16. Jonsson F, Goicolea I. "We believe in you, like really believe in you": Initiating a realist study of (re)engagement initiatives for youth not in employment, education or training with experiences from northern Sweden. Evaluation and Program Planning. 2020; doi:

10.1016/j.evalprogplan.2020.101851.

17. Simmons R, Thompson R. Reclaiming the disengaged: critical perspectives on young people not in education, employment or training. Research in Post-Compulsory Education. 2013; doi: 10.1080/13596748.2013.755799.

18. Raposa EB, Rhodes J, Stams GJJM, Card N, Burton S, Schwartz S, et al. The Effects of Youth Mentoring Programs: A Meta-analysis of Outcome Studies. Journal of Youth and Adolescence. 2019; doi: 10.1007/s10964-019-00982-8.

19. Hermens N, Super S, Verkooijen KT, Koelen MA. A Systematic Review of Life Skill Development Through Sports Programs Serving Socially Vulnerable Youth. Research Quarterly for Exercise and Sport. 2017; doi: 10.1080/02701367.2017.1355527

20. Nelson J, O'Donnell L. Approaches to Supporting Young People Not in Education, Employment or Training: a Review (NFER Research Programme: From Education to Employment). Slough: NFER; 2012.

21. Mawn L, Oliver EJ, Akhter N, Bambra CL, Torgerson C, Bridle C, et al. Are we failing young people not in employment, education or training (NEETs)? A systematic review and meta-analysis of reengagement interventions. Systematic Reviews. 2017; doi: 10.1186/s13643-016-0394-2

22. Rajasekaran S, Reyes J. Back to school: Pathways for reengagement of out-of-school youth in education. Washington, DC: The World Bank; 2019.

23. Russell $L$, Simmons R, Thompson R. Playing the numbers game: Connexions personal advisers working with learners on entry to employment programmes. Journal of Vocational Education \& Training. 2010; doi: 10.1080/13636820903427561.

24. Pawson R. The science of evaluation: a realist manifesto. Thousand Oaks, CA: Sage; 2013.

25. Wong G, Westhorp G, Manzano A, Greenhalgh J, Jagosh J, Greenhalgh T. RAMESES II reporting standards for realist evaluations. BMC Medicine. 2016; doi: 10.1186/s12916-016-0643-1. 
26. Hoggarth L, Smith DI. Understanding the Impact of Connexions on Young People at Risk. London: Department for Education and Skills; 2004.

27. Owen E. A series of realist evaluations of multi-component programmes with disengaged young people: What works, for whom, and in what contexts? Swansea: Swansea University; 2021.

28. Kane M, Trochim WM. Concept mapping for planning and evaluation. Thousand Oaks, CA: Sage; 2007.

29. Swedish Institute. Education in Sweden, Stockholm: Swedish Institute; 2021 [Available from: https://sweden.se/society/education-in-sweden/

30. Swedish Higher Education Authority. Higher education institutions in Sweden - 2020 status report. Johanneshov: UK ̈̈; 2020.

31. Pawson R, Tilley N. Realistic Evaluation. London: Sage; 1997.

32. Lacouture A, Breton E, Guichard A, Ridde V. The concept of mechanism from a realist approach: a scoping review to facilitate its operationalization in public health program evaluation. Implementation Science. 2015; doi: 10.1186/s13012-015-0345-7

33. The RAMESES II Project. What is a mechanism? What is a programme mechanism? 2017.

34. Dalkin SM, Greenhalgh J, Jones D, Cunningham B, Lhussier M. What's in a mechanism? Development of a key concept in realist evaluation. Implementation Science. 2015;doi: 10.1186/s13012-015-0237-x.

35. Jagosh J, Macaulay AC, Pluye P, Salsberg JON, Bush PL, Henderson JIM, et al. Uncovering the Benefits of Participatory Research: Implications of a Realist Review for Health Research and Practice. The Milbank Quarterly. 2012; doi: 10.1111/j.1468-0009.2012.00665.x.

36. Jagosh J. Realist Synthesis for Public Health: Building an Ontologically Deep Understanding of How Programs Work, For Whom, and In Which Contexts. Annual Review of Public Health. 2019; doi: 10.1146/annurev-publhealth-031816-044451.

37. Jagosh J, Pluye P, Wong G, Cargo M, Salsberg J, Bush PL, et al. Critical reflections on realist review: insights from customizing the methodology to the needs of participatory research assessment. Research synthesis methods. 2014; doi: 10.1002/jrsm.1099.

38. Windsor LC. Using Concept Mapping in Community-Based Participatory Research: A Mixed Methods Approach. Journal of Mixed Methods Research. 2013; doi: 10.1177/1558689813479175

39. Manzano A. The craft of interviewing in realist evaluation. Evaluation. 2016; doi: $10.1177 / 1356389016638615$

40. Pawson R. Evidence-based Policy: The Promise of 'Realist Synthesis'. Evaluation. 2002; doi: $10.1177 / 135638902401462448$

41. Rycroft-Malone J, McCormack B, Hutchinson AM, DeCorby K, Bucknall TK, Kent B, et al. Realist synthesis: illustrating the method for implementation research. Implementation Science. 2012; doi: 10.1186/1748-5908-7-33

42. Pawson R. Evidence-based policy: a realist perspective. London: Sage; 2006. 
43. Saul JE, Willis CD, Bitz J, Best A. A time-responsive tool for informing policy making: rapid realist review. Implementation Science. 2013; doi: 10.1186/1748-5908-8-103

44. Pawson R, Greenhalgh T, Harvey G, Walshe K. Realist review - a new method of systematic review designed for complex policy interventions. Journal of Health Service Research \& Policy. 2005; doi: $10.1258 / 1355819054308530$

45. Wong G, Greenhalgh T, Westhorp G, Buckingham J, Pawson R. RAMESES publication standards: realist syntheses. BMC Medicine. 2013; doi: 10.1186/1741-7015-11-21

46. Wynn DE, Williams CK. Recent advances and opportunities for improving critical realism-based case study research in IS. Journal of the Association for Information Systems. 2020; doi:

10.17705/1jais.00592

47. Pawson R. Theorizing the Interview. The British Journal of Sociology. 1996; doi: 10.2307/591728

48. Wiltshire G, Ronkainen N. A realist approach to thematic analysis: making sense of qualitative data through experiential, inferential and dispositional themes. Journal of Critical Realism. 2021: doi: 10.1080/14767430.2021.1894909

49. Aaltonen A, Tempini N. Everything Counts in Large Amounts: A Critical Realist Case Study on DataBased Production. Journal of Information Technology. 2014; doi: 10.1057/jit.2013.29

50. Yin RK. Case study research: design and methods. 4 ed. Thousand Oaks: Sage; 2013.

51. Aldamman K. Managerial Practices to Ensure the Well-being of Humanitarian Volunteers: A Realist Evaluation. Dublin: Trinity College Dublin; 2020.

52. Mills AJ, Durepos G, Wiebe E. Encyclopedia of Case Study Research. Thousand Oaks: Sage; 2010.

53. Emmel N. Sampling and Choosing Cases in Qualitative Research: A Realist Approach. London: SAGE; 2013.

54. Clark A, Holland C, Katz J, Peace S. Learning to see: lessons from a participatory observation research project in public spaces. International Journal of Social Research Methodology. 2009; doi: $10.1080 / 13645570802268587$

55. Bowen GA. Document Analysis as a Qualitative Research Method. Qualitative Research Journal. 2009; doi: 10.3316/QRJ0902027

56. Concept Systems. Concept Systems Software. Ithaca, NY: Concept Systems, Incorporated; 2013.

57. WMA. World Medical Association Declaration of Helsinki. Ethical Principles for Medical Research Involving Human Subjects. Seoul: WMA General Assembly; 2008.

58. Atkins L. Researching 'with', not 'on': engaging marginalised learners in the research process. Research in Post-Compulsory Education. 2013; doi: 10.1080/13596748.2013.755853

59. Conolly A. Challenges of Generating Qualitative Data with Socially Excluded Young People. International Journal of Social Research Methodology. 2008; doi: 10.1080/13645570701401446

60. Russell L. Researching marginalised young people. Ethnography and Education. 2013; doi: $10.1080 / 17457823.2013 .766433$ 
61. Goicolea I, Carson D, San Sebastian M, Christianson M, Wiklund M, Hurtig A-K. Health care access for rural youth on equal terms? A mixed methods study protocol in northern Sweden. International Journal for Equity in Health. 2018; doi: 10.1186/s12939-018-0718-z

62. Jonsson F, Goicolea I, Christianson M, Carson DB, Wiklund M. Landscapes of care and despair for rural youth - a qualitative study in the northern Swedish 'periphery'. International Journal for Equity in Health. 2020; doi: 10.1186/s12939-020-01288-z

63. Lögdberg U, Nilsson B, Kostenius C. "Thinking about the future, what's gonna happen?" How young people in Sweden who neither work nor study perceive life experiences in relation to health and wellbeing. International Journal of Qualitative Studies on Health and Well-Being. 2018; doi: 10.1080/17482631.2017.1422662

64. Smyth J, Mclnerney P, Fish T. Re-engagement to where? Low SES students in alternative-education programmes on the path to low-status destinations? Research in Post-Compulsory Education. 2013; doi: $10.1080 / 13596748.2013 .755862$

65. Nairn K, Higgins J. The emotional geographies of neoliberal school reforms: Spaces of refuge and containment. Emotion, Space and Society. 2011; doi: 10.1016/j.emospa.2010.10.001

66. MacDonald R, Marsh J. Disconnected Youth? Journal of Youth Studies. 2001; doi: $10.1080 / 13676260120101860$

\section{Supplementary Files}

This is a list of supplementary files associated with this preprint. Click to download.

- Additionalfile1.docx 